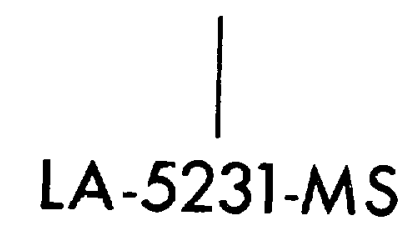

INFORMAL REPORT

\section{An Encomium on Solar Sailing}

THIS DOCUMENT CONFIRMED AS

UNCLASSIFIED

DIVISION OF CLASSIFICATION

BY I.L.Cuice hiciralwer
DATE $6 / 18 / 23$

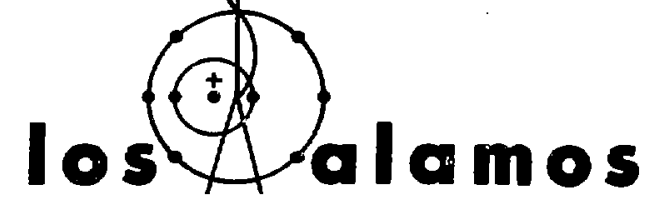

scientific laboratory

of the University of California

los alamos, NEW MEXICO 87544 
This report was prepared as an account of work spc nsored by the United States Government. Neither the United States nor the United States Atomic Energy Commission, nor any of their employees, nor any of their contractors, subcontractors, or their employees, makes any warranty, express or implied, or assumes any legal liability or responsibility for the accuracy, com. pleteness or usefulness of any information, apparatus, product or process disclosed, or represents that its use would not infringe privately owned rights.

This informal report was originally issued in $1958 \mathrm{as}$ an office memorandum. Continuing interest in this topic has prompted issuance in the LA-series form to allow wider distribution.

Printed in the United States of America. Available from National Technical Information Service

U. S. Department of Commerce 5285 Port Royal Road

Springfield, Virginia -2151

Price: Printed Copy $\$ 300 ;$ Microfiche $\$ 0.95$

$+4 . \infty$ 
LA-5231-MS

Informal Report

UC-33

ISSUED: May 1973

\section{scientific laboratory \\ of the University of California \\ LOS ALAMOS, NEW MEXICO 87544}

1

\section{An Encomium on Solar Sailing}

by

T. P. Cotter

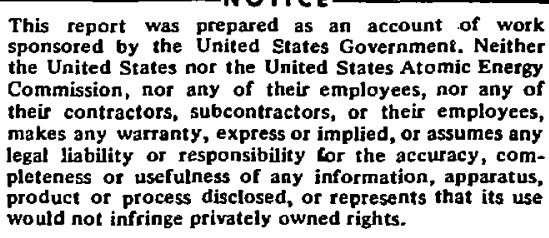
would not infringe privately owned rights. 
AN ENCOMIUM ON SOLAR SAILING

\author{
by \\ T. P. Cotter
}

The ldea of using the pressure of solar radiation for extraorbital propulsion of rather circumscribed performasce has been considered and apparently depreclated often before. There are, however, two independent articles $(1,2)$ describing similar conceptions of solar salling vehicles which are physically sound, quantitatively correct and extremely promising. What "Russeli Saunders" and R. L. Garwin each have done is to recognize and describe methods of manipulating a radiation-propelled sail of what appears to be practical mechanical character so as to travel essentially at will throughout the solar system, achieving quite reasonable flight times for a broad category of int - -esting interplaretary missions.

The present note contains little new beyond the observations of the previous authors, except the notion of spinning the sail. Its intent is to advertise the considerable merits of solar salling by filling in more details of the scheme. In order to expose the problems and indicate some technical possibilities for their solution, I will presently describe the construction, operation and flight of an unmanned instrumented solar sailing vericle on a round trip of exploration to the neighborhood of lars under commsnd guidance from the earth. Before presenting this particular body of circumstantial evidence in favor of feasibility it seems worthwhile to provide some incentive for the task by emphasizing some of the implications of the properties of solar-sailing vehicles in general.

A solar sailing vehicle cannot take off from the surface of any substantial gravitating body, but must be launched into orbit by other means. It may, however, contain a re-entry body for landing through an atmosphere. A conservatively designed solar sail can have an acceleration of greatex than $0.1 \mathrm{~cm} / \mathrm{sec}^{2}$ in the radiation field at the earti's distance from the sun. This is already significantly better than present optimistic

(1) Russell Saunders, "Astounding Science Fiction", pp 136-143, May 1951.

(2) R. L. Garwin, "Jet Propulsion" 8, 188(1958). 
hopes for nuclear-powered systems of very high specific impulse and small mass ratio. The implications of this for those of us who have been thinking about nuclear milli-g devices are severe, at least for flight in the near solar system, say inside the orbit of Jupiter. An upper limit on the possible acceleration of a solar sail is imposed by the penetration of radiation through thin metallic fllms, and this acceleration is of the order of magnitude of $5 \mathrm{~cm} / \mathrm{sec}^{2}$ at the earth's solar distance.

The radiation-produced thrust of a solar sail is not arb1trary in direction, for it may not have a component toward the sun. The reflection of light from a sail surface oriented at an angle to the incident radiation can, however, provide a component of force perpendicular to the solar direction. Since a sunward force is inevitably supplied by solar gravitational attraction, the limitation on thrust direction does not imply a restriction on the ability of a solar sall to travel between arbitrary points in interplanetary space. This ability lies essentialiy in the fact that one can use the sall to provide a non-central force component so as to alter the angular momentum of the vehicle about any gravitating body. Roughly speaking, this permits one to alter the balance between centrifugal force and gravitational attraction in favor of either one. It is pleasant to note also that the limitation in thrust direction does not Impose a large penalty on the time required to travel from point to point.

The mass of a solar sailing vehicle is arbitrary. At the small end of the scale, a vehtcle of mass not exceeding a few kilograms could carry a programmed mechanism which would cause it to maneuver so as to escape from an orbit about the earth. Vehicles of large payload principally involve the mechanical problem of manipulating rather large areas of sail. For example, a vehicle with a payload of 10,000 kilograms might employ a sail area of the order of 10 square kilometers, which is equivalent to a fleet of a thousand clipper ships, more-or-less.

At the present time we have the capability of placing modest sateliftes in orbit. Te could therefore begin now in the actual practice of the rudiments of this technique of interplanetary fight. Concurrently with our abllity to orbit heavier objects, the solar salling vehtcles could grow in sophistication and usefulness.

The really striking propertles of solar sailing vehicles are consequences of the fact that they roquire no propeliant. The useful prorulsive effort that can be obtained from a solar sail is determined only by its freedom from malfunction and wear. In addition to employment as vehicles for long-sustained interplanetary exploration, one may envision for example, a solar sail in repeated use for maneuvering up to objects which have been placed in sateliite orbit and transporting thes elsewhere. 
The boundaries of the range of action of solar sailing vehicles are roughly determined by two practical considerations. The temperature and speed of a solar sall would be comparable respectively to the surface temperature and orbital velocity of a planet at the sails distance from the sun. This means that a solar sailing vehicle encounters high temperature problems inside the orbit of Mercury, and requires many years for interplanetary flight in the outer region of the solar system.

The indefinitely sustained thrust property of the solar sall greatly simplifies the problem of navigational guidance. Variable thrust is always available for course correction throughout a journey. The penalty for a non-optimum trajectory, or an actual navigational exror is only to the flight time, which is hardly the case for a rocket operating with a small fuel margin.

Wide variations in the design of solar sailing vehicles are permitted because their parts are subject only to mild stresses, they are not required to perform mechanically difficult operations and they do not experience strongly hostile environments. For this reason the details of the particular Mars-exploring vehicle which will now be described are largely arbitrary. They are simply illustrative of a possible compatible set of properties. I have chosen to consider a vehicle of rather more sophistication than would be attempted initially in orcer to emphasize what may be done with a mass which we will be able to place in satellite orbit within a few years.

The vehicle as prepared for launching would be contained In a capsule of a few cubic meters volume and weighing $500 \mathrm{Kg}$. of this mass, $250 \mathrm{Kg}$ is devoted to the sail, a flat circular disk $10^{-4} \mathrm{~cm}$ thick and 500 meters in diameter, made of plastic film coated on one side with 20 or $30 \mathrm{micrograms} / \mathrm{cm}^{2}$ of aluminum. While this plastic film is fragile by ordinary structural standards, it can readily be handled for fabrication, folding and packing into the launching capsule, for a strip of the material $1 \mathrm{~cm}$ wide can be expected to bear a load of 100 grams in tension without rupture. To stop the propagation of any tears which develop in use the sall is made in panels with reinforced seams. The sall material required here has about one-third the thickness of plastic film now available commercially.

The remaining $250 \mathrm{Kg}$ of load consists of light structural members, solar batteries, radio transmitter-recelver, directional antenna, control servomechanisms, navigational equipment and data gathering instrumentation.

For convenience the vehicle is launched into a circumpolar satelifte orbit at sunrise or sunset so it will be continuously in sunlight thereafter. If the orbit perigee exceeds $1500 \mathrm{~km}$ then the aerodynamic drag force will be less than $0.1 \%$ of the 
radiation pressure force when the sail is spread. The vehicle has a maximum available acceleration of $0.3 \mathrm{~cm} / \mathrm{sec}^{2}$, whlch $1 \mathrm{~s}$ one-half the acceleration due to solar gravitation at the earth orbital radius.

The capsule consists of two parts connected through a universal joint, and provided with a motor which now causes the sail package and the main part of the load to start counterrotating. At the appropriate times the collapsed structural backbone is extended, the capsule cases are jettisoned and the vehicle blossoms forth under centrifugal force to assume the configuration shown schematically in Figure 1. Approximate dimensions are given. The sail is spinning at the rate of one revolution in two minutes. The load, two-thirds of which is suspended in three pods by wires at $\mathbf{5 0}$ meters from the axis of spin, is counter-rotating at a rate of 10 revolutions per minute. The vehicle as a whole has zero net angular momentum.

As well as serving to spread the sall, the spin holds it nearly flat. Under the combined action of radiation pressure and centrifugal force the sail assumes the shape of a wide exponential horn with a diameter of 500 meters and a length of less than 5 meters. The stress in the sail nowhere exceeds $0.1 \%$ of the breaking strength of the plastic.

The rotation serves another important function. It provides a straightforward means of orienting the vehicle in space as desired, without the aid of external forces. A torque acting between the load and sail axial spars, as exemplified by the tilt actuator of Figure 1 , will cause both to precess gyroscopically. The reaction to the torque on the sail is a counter torque on the load, which causes it to precess in such a way as to conserve the zero angular momentum of the whole vehicle, the load and sail axial spars remaining approximately colinear. There are two orthogonal angular degrees of freedom, the axis of precessional rotation and the amount of precession, so that the vehicle can in fact be turned by this means from any arbitrary orientation in space to any other. The details of the turning as far as the sail is concerned are rather compl1cated, as they involve the propagation of waves of deflection from the ring at which the guy strips are attached out across the centrifugally stressed sail. Neglecting problems of vibrational resonance which may arise, the velocity of rotation and the placement of the guy strips have been chosen for the present sail so that the vehicle may be precessed through one revolution in two hours. Not coincidentally, this is also the orbital period of a $1500 \mathrm{~km}$ altitude earth satelite.

For communication of data and Instructions between the vehicle and earth one might employ beamed pulse telegraphy with an information rate of the order of one bit per second. If the 
vehtcle has an antenna area of 10 square meters and the earth station one of 500 square meters, a vehicle transmitter power of less than 100 waits will be sufficient for the Mars filght.

For astrogation one requires to know the position and orientation of the vehicle. Two light detection systems, and the communications beam itself might serve for this. One ilght detector would lock on the sun and report its angle with respect to the sail spin axis. The second 1 ight detector would scan the celestial sphere and report the direction and angular magnitude of the brightest object other than the sun. The detector will not be required to locate anything smaller than one-tenth the visual size of the moon. This pair of detectors is used to establish the position and orientation of the vehicle when near fither the earth or another planet.

Ixcept near the earth, the vehicle antenna is servo-controlled to point at the earth transmitter. This, together with the solar 1 ight detector, provides internal reference axes for the vehicie when far from any planet. The direction of the earth antenna and the transit time of the communications signal between earth and vehicle can establish position only coarsely but still accurately enough to place the vehicle in a xegion of space near the terminal planet, where the planetary guidance system can function effectively.

There are conflgurations in which this astrogation system does not uniquely define position or orientation. One may elect to coast through such configurations, or perhaps simply avold them.

In the description of salling maneuvers to follow, a convention $w i 11$ be adopted to avoid repetitlous locution. The sail angle is said to be positive if the sail force has a component in the same direction as the component of vehicle velocity perpendicular to the solar radius vector, and is negative if the sail force has a component opposed to the same vehicle velocity component.

The vehicle, which is now orbiting the earth in a plane perpendicular to the solar radius vector, escapes from the earth's gravitational field by orientine the sail so that a prolonged diameter of the sall passes through the center of the earth, and the sail spin axis is maintained at an angle of plus 350 (arc sin $\sqrt{3 / 3}$ ) with respect to the solar direction. This will cause the vehicle to spiral away from the earth pith the sail precessing through one revolution of turn for each orbital revolution. In two months the vehicle arrives in a region where the earth's and sun's gravitational forces on the vehicle are the same order of magnitude. In another 10 days the vehicle has efiectively escaped from the earth and is an independent body orbiting about the sun. 
The time is now about 20 days before the opposition of earth and Mars. The sall is set so that the spin axis 1 ies in the plane of the ecliptic and continually makes an angle of minus $12^{\circ}$ with the solar direction. At the end of 2 months the latter angle is changed to plus $60^{\circ}$. In a bit more than 8 months the vehicle arrives in the vicinity of Mars, tangent to its orbit and with the same velocity as Mars. This trajectory, and the later return to earth is shown in Figure 2.

The general problem of specifying the maneuver which will carry a given solar sail from one planet to another in the minimum time appears to be fairly difficult. In order to exhibit a set of sailing directions which will actually accomplish the desired mission, I have resorted to graphical interpolation among a set of machine computed numerical integrations of the equations of motion for the solar sail. Of all the one-parameter family of orbits with no more than two sail angle settings, the one given yields the minimum time of transit between earth and Mars. Orbital ellipticity and inclination were neglected in these calculations.

The vehicle now has $6-1 / 2$ months available before the first favorable time for its return to earth. This is sufficient for it to spiral down to a close satellite orbit about Mars and escape again.

The return to earth is accomplished by setting the sail spin axis in the plane of the ecliptic and making an angle of minus $60^{\circ}$ with the solar direction for a little more than 8 months, followed by a setting of plus $12^{\circ}$ for 2 months. At the end of this time the vehicle is in the vicinity of earth. Slightly less than $2-1 / 2$ years have elapsed from the moment of launching.

Some of the hazards of space should be mentioned. Plastic films deteriorate in strong ultraviolet radiation. However, the sails' aluminum reflecting layer is nearly opaque, and there is no maneuver which requires facing the plastic side of the sail toward the sun.

A rough notion of the rate of meteoritic attrition of the sail can be based on current estimates of the density of micrometeorites in interplanetary space. One arrives at the impresslon that the half-life for decay of sail reflectivity will be measured in thousands of years.

Any remarks on the effects of interplanetary electromagnetic fields would be purely conjectural. These are typical of questions which are perhaps best answered by sending out a solar sail to see. 


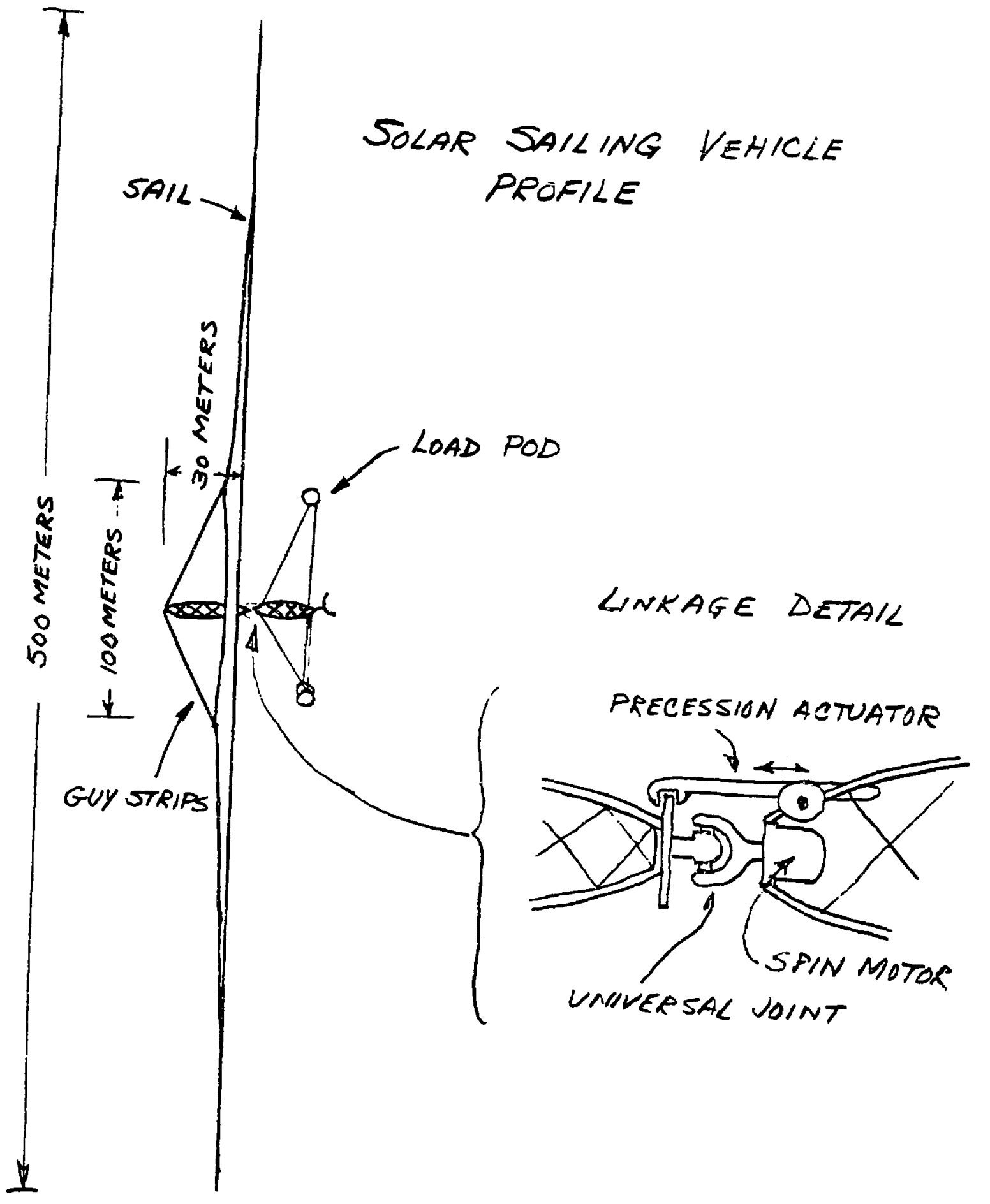

Figure 1 


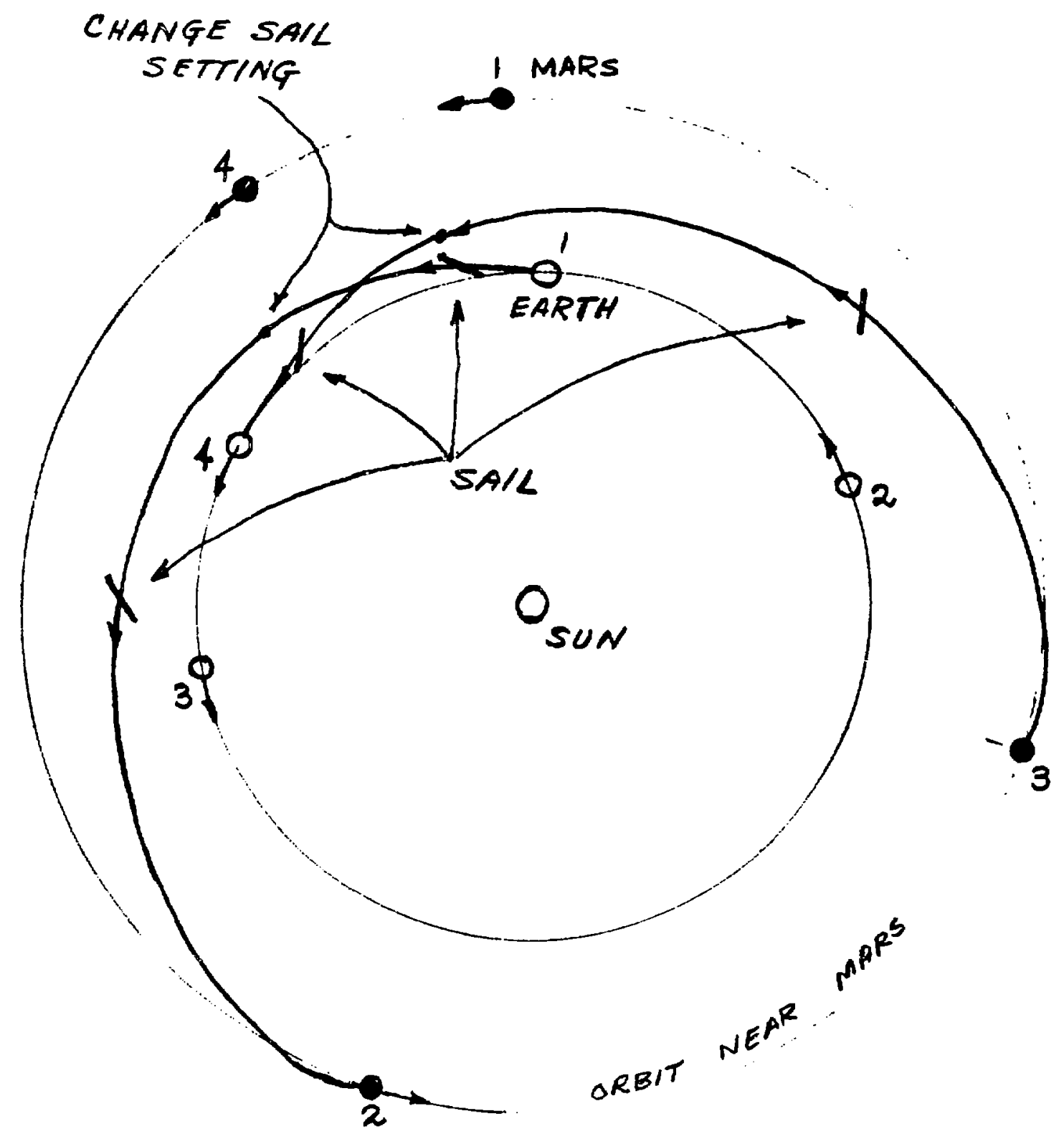

FIGURE Z - SOLAR SAILING ORBITS BETWEEN EARTH AND MARS (EQUAL TIME POSITIONS ARE SIMILARLY NUMBERED, IN SEQUENCE.)

CM: $267(60)$

8 